\title{
.. F.'Ingenierías
}

\section{Atendimento educacional especializado e as demandas de tecnologia assistiva nas práticas inclusivas brasileiras}

\section{Helena Sardagna}

Professora Curso de Pedagogia, Grupo de Estudos em Educação e Processos Inclusivos, Universidade Estadua do Rio Grande do SUI (UERGS), Porto Alegre, Rio Grande do Sul, Brasil. helena-sardagna@uergs.edu.br.

Recibido: 15 Ago 2016 - Revisado: 30 Sep 2016 Aceptado: 30 Oct 2016 - Publicado: 30 Dic 2016

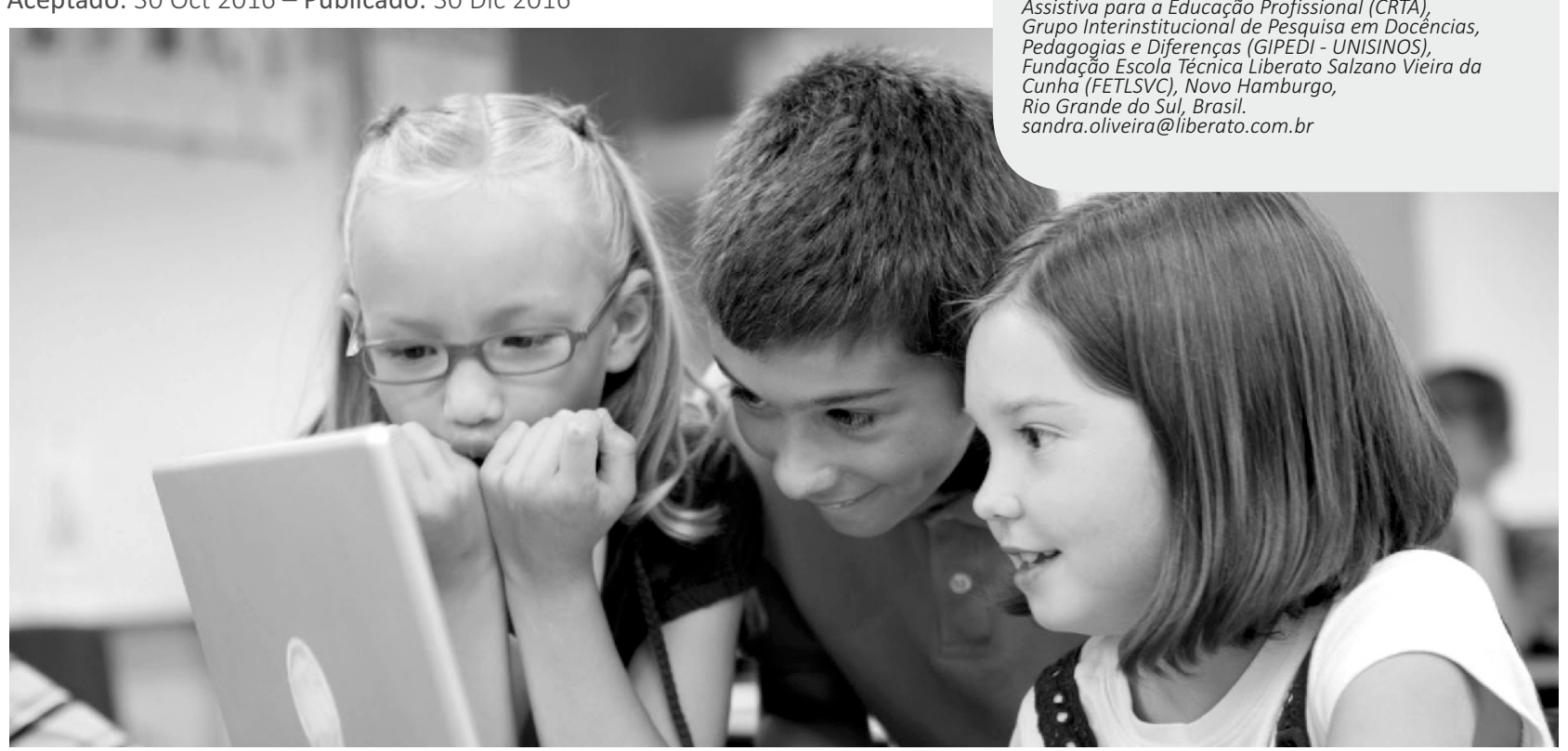

Resumo: O presente artigo apresenta uma análise que visou identificar demandas de tecnologia assistiva para a criação de banco de dados virtual, a partir de levantamento junto a professores de Atendimento Educacional Especializado (AEE) em escolas públicas de dois municípios da região metropolitana de Porto Alegre/RS- Brasil. O texto transita pelos argumentos da inclusão e da acessibilidade, especialmente na produção de Tecnologia Assistiva (TA) para o AEE, visando à inserção e permanência das pessoas com deficiência, tanto na escola, quanto na formação profissional com vistas ao mercado de trabalho. Como resultado, a pesquisa apontou para sete demandas de TA e a criação de banco de dados virtual, disponível na web.

Palavras-chave: atendimento educacional especializado; banco de dados; educação inclusiva; tecnologia assistiva.

Abstract: This article proposes an analysis aimed at identifying the demands of assistive technology for the virtual database creation, from survey with ESA teachers in public schools in two municipalities in the metropolitan region of Porto Alegre / RS- Brazil. The text moves by the inclusion and accessibility arguments, especially in the production of Assistive Technology (AT) for Educational Specialized Care (AEE), aiming at the inclusion and permanence of people with disabilities, both in school and in vocational training with a view to the labor market. As a result, the survey pointed to seven demands of TA and the virtual database creation, available on the web.

Keywords: specialized educational services; database; inclusive education; assistive technology. 


\section{INTRODUÇÃO}

$\mathrm{Na}$ atualidade, a bandeira da inclusão é levantada em todas as instâncias da sociedade, no discurso da educação como um direito humano. Nessa perspectiva, incluir alunos na rede regular de ensino é proporcionar aos sujeitos a garantia de acesso à escolarização, da efetivação de permanência na escola e da garantia de aprendizagem inclusive na educação profissional (MEC 1996; Conselho Nacional de Educação 2009; Casa Civil 2013). No entanto, essa premissa só irá se efetivar se a pessoa com deficiência tiver contemplado o direito à educação com igualdade de condições.

Essa perspectiva vem sendo defendida para todos os níveis e modalidades de ensino. Entretanto, dados da Organização Mundial da Saúde (OMS) (2012), apontam para um hiato no que diz respeito ao ensino médio e à educação profissional, pois a empregabilidade desejada não tem ocorrido. Essas lacunas têm mobilizado instituições governamentais e não-governamentais a criarem estratégias para promover as condições e tornar concreta a inclusão escolar e a acessibilidade, a exemplo de instrumentos como a Política Nacional de Educação Especial na Perspectiva da Educação Inclusiva (MEC/SEESP 2008; Conselho Nacional de Educação 2009), o Plano Viver sem Limites (SDH/SNPD 2011); a Lei Brasileira da Pessoa com Deficiência, Lei 13.146 de 2015, entre outros. Esses documentos propõem o Atendimento Educacional Especializado como apoio à inclusão para "pessoas com deficiência, transtorno global do desenvolvimento e altas habilidades/superdotação" [4].

Essa problematização serviu como pano de fundo para uma pesquisa realizada em 2015, que objetivou identificar demandas de tecnologia assistiva para inserção em banco de dados virtual, envolvendo quatro docentes pesquisadores e três alunos bolsistas de iniciação científica, tendo como população-alvo professores de Salas de Recursos Multifuncionais de dois municípios localizados na região metropolitana de Porto Alegre. A pesquisa possibilitou reunir dados que indicam demandas de tecnologia assistiva, nas salas de recursos pesquisadas, para que a proposta da inclusão escolar se efetive. O instrumento de coleta de dados foi questionário eletrônico on line semiestruturado, com questões objetivas e abertas.

\section{ATENDIMENTO EDUCACIONAL ESPECIALIZADO: DEMANDAS DE TECNOLOGIA ASSISTIVA}

O conceito de Tecnologia Assistiva (TA) é empregado, nesse estudo, a partir da definição dada pelo o Comitê de Ajudas Técnicas (CAT), instituído pela Portaria $\mathrm{n}^{\circ}$ 142, de 16 de novembro de 2006, que define a TA como uma área do conhecimento, de característica interdisciplinar, que engloba produtos, recursos, metodologias, estratégias, práticas e serviços que objetivam promover a funcionalidade, relacionada à atividade e participação de pessoas com deficiência, incapacidades ou mobilidade reduzida, visando sua autonomia, independência, qualidade de vida e inclusão social (Subsecretaria Nacional de Promoção dos Direitos da Pessoa com Deficiência 2014).

A TA é sugerida nas ações de apoio à inclusão, como forma de garantir à pessoa com deficiência acesso a recursos que maximizem sua autonomia, mobilidade pessoal e qualidade de vida.

Observa-se uma carência de conhecimento sobre a TA, como uma proposição presente na política atual da educação especial, na perspectiva da educação inclusiva. Bersch (2015) enfatiza que há insuficiência de informações fundamentais sobre Tecnologia Assistiva, tanto para os usuários, quanto para os professores que promovem a TA. Tais informações estão restritas a agentes governamentais, e acessíveis a poucos profissionais das áreas da saúde, educação, assistência social, direitos humanos, entre outros. 
As informações da pesquisa, juntamente com outras duas pesquisas desenvolvidas no mesmo período, uma com foco em cursos da educação profissional e outra em empresas e indústrias, compõem um banco de dados para pesquisadores da área de TA, contribuindo para que novas investigações e soluções tecnológicas possam ser desenvolvidas. A busca por identificar demandas de Tecnologia Assistiva em Salas de Recursos Multifuncional também visa contribuir para a qualificação das práticas inclusivas na região, bem como para a inserção dos sujeitos com deficiência no ensino médio e educação profissional e, consequentemente, no mercado de trabalho.

Proposta de banco de dados foi encontrada com o Solassist - Biblioteca Virtual de Soluções Assistivas composto por soluções assistivas. De importante relevância no Brasil o banco também é chamado de biblioteca de sugestões assistivas e é desenvolvido no âmbito do projeto Solassist, pelo grupo de pesquisa Tecnologia em Educação para Inclusão e Aprendizagem em Sociedade (TEIAS), da Universidade Federal do Rio Grande do Sul (UFRGS). A proposta na pesquisa aqui descrita, diferentemente do banco de dados da UFRGS, tem como objetivo principal disponibilizar problemas/demandas de TA.

O Relatório Mundial sobre a deficiência (OMS 2012) aponta uma lacuna que precisa ser suprida, no que tange à empregabilidade dos jovens com deficiência justamente por falta de oportunidades de formação que contemple suas habilidades e competências. Neste sentido, "educação e treinamento são primordiais para um trabalho bom e produtivo" (MEC/SEESP 2008).

As instituições são orientadas a criarem espaços de inclusão no ensino médio e educação profissional, com perspectivas de inserção no mercado de trabalho, no entanto, a promoção dessas práticas ainda é um grande desafio para a educação profissional.
Historicamente as pessoas com deficiência foram excluídas dos espaços públicos, das escolas, do mercado de trabalho e da convivência em sociedade. Nesse contexto a pessoa com deficiência era responsável por adaptar-se para viver em sociedade, porém, no modelo inclusivo, cabe à sociedade adaptar-se para acolher as diferenças e promover condições de acesso e todos espaços sociais (Garcia 2008). O auxílio da Tecnologia Assistiva (TA) é uma solução possível para essa problemática, uma vez que integra diversas áreas do conhecimento, como psicologia, arquitetura, engenharia, fisioterapia, pedagogia, entre outras.

As lacunas encontradas nas instituições de educação, em relação às TA, apontam a necessidade de se intensificar e incentivar a sua produção. O Estatuto da Pessoa com Deficiência prevê a criação de mecanismos de fomento à pesquisa, prevendo, inclusive, a concessão de linhas de crédito subsidiado pelo poder público, em parcerias com institutos de pesquisa oficiais (Casa Civil 2015).

Essa demanda também está contemplada no Plano Viver sem Limite da Secretaria Nacional de Promoção dos Direitos da Pessoa com Deficiência (SDH/SNPD 2011), que prevê programa de inovação em Tecnologia Assistiva, atendimento educacional especializado, crédito para aquisição de produtos de tecnologia assistiva, entre outros.

O referido Plano sugere a implantação de espaços equipados com materiais pedagógicos para oferta do atendimento educacional especializado, denominados Salas de Recursos Multifuncionais, tal como prevê o documento da Política Nacional de Educação Especial na perspectiva da Educação Inclusiva (MEC/SEESP 2008). Junto a este eixo está o Programa Nacional de Acesso ao Ensino Técnico e Emprego (PRONATEC), que assegura o ingresso de pessoas com deficiência, independentemente do ofertante, do curso e do tipo de deficiência, com 
atendimento preferencial na ocupação das vagas (SDH/SNPD 2011).

\section{RESULTADOS}

A investigação teve foco nas demandas e necessidades de tecnologia assistiva das escolas públicas de educação básica que possuem Sala de Recursos Multifuncional nos municípios de Novo Hamburgo e São Leopoldo, para promoção do atendimento educacional especializado de pessoas com deficiência. A finalidade da pesquisa foi criar mecanismos para incentivar as pessoas com deficiência a continuarem seus estudos em níveis mais elevados de estudo, inclusive na educação profissional. O resultado da pesquisa contribuiu com a construção de um banco virtual de demandas de tecnologia assistiva que visa estimular o desenvolvimento de soluções tecnológicas e metodológicas para a inclusão de alunos com deficiência no ensino médio e educação profissional.

Os dados foram levantados por meio de formulário criado a partir do serviço Google Drive (ferramenta para criação de formulário de coleta de dados, disponível em https://drive.google.com) e enviado ao endereço eletrônico disponibilizado pelos professores de Salas de Recursos Multifuncionais (SRM) ou profissionais responsáveis pelas escolas participantes da pesquisa. Receberam o convite para participar 65 professores de SRM, porém, cerca de $50 \%$ deram retorno. Entre as escolas participantes, 4 \% não possui professor específico que atua na SRM. As mesmas atendem deficiências variadas, porém, percebe-se uma predominância no atendimento da deficiência intelectual, com cerca de $96 \%$.

A pesquisa indica que $69 \%$ das escolas pesquisadas utilizam algum tipo de tecnologia nos seus atendimentos, tais como softwares, protetor de teclado (colmeia), leitor de tela ou mouse adaptado. São citadas também algumas tecnologias construídas de forma artesanal.
Dentre os itens de Tecnologia Assistiva citados no questionário para atender ao público-alvo das Salas de Recursos Multifuncionais existentes, estão (a) jogos online, com comando sem mouse, implementados de maneira mais atrativa do que os já existentes; (b) materiais acessíveis para a educação física; (c) tesoura adaptada de baixo custo; (d) cadeira de rodas própria para educação física de baixo custo; (e) softwares que auxiliem no aprendizado da leitura e escrita e que trabalhem noções matemáticas; (f) ferramentas que auxiliem na alfabetização dos alunos autistas e deficientes intelectuais; (g) equipamentos que estimulem a fala e a emissão de sons, entre outros. Os dados ainda apontaram para a dificuldade que as escolas têm de contar com profissionais de diferentes áreas do conhecimento como informática, mecânica, eletrônica, entre outras, para a produção de tecnologia.

Fica evidente a necessidade de produzir TA para apoio à inclusão escolar e social com foco na autonomia e independência, visando à qualidade de vida das pessoas com deficiência, tal como preconizam as orientações do Comitê de Ajudas Técnicas, da Subsecretaria Nacional de Promoção dos Direitos das Pessoas com Deficiência (Subsecretaria Nacional de Promoção dos Direitos da Pessoa com Deficiência 2014).

Como resultado a investigação aqui apresentada, "Pesquisa e produção de tecnologia assistiva: uma parceria entre a educação básica e o ensino superior", constituiu um conjunto de demandas em tecnologia assistiva para o atendimento educacional especializado. Essas demandas foram inseridas em banco de dados intitulado "Observatório de demandas em TA do Centro de Referência em Tecnologia Assistiva para a Educação Profissional" da Fundação Liberato. 


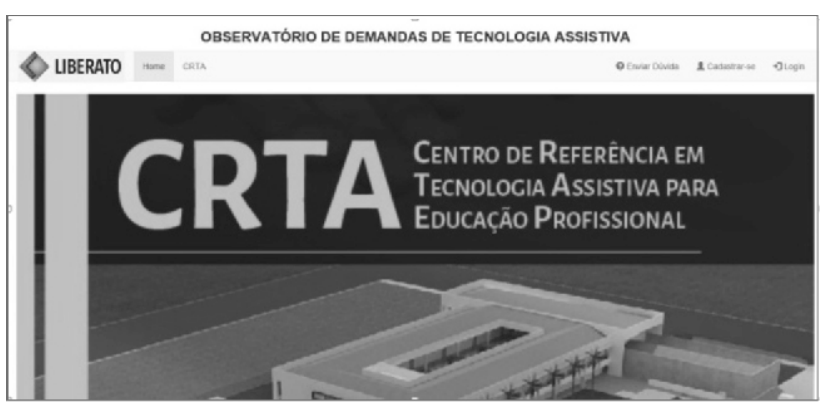

Figura 1. Tela inicial do Observatório de demandas em TA - Chave de busca para demandas em TA.

Disponibilizado virtualmente pelo Centro de Referência, o banco tem como objetivo divulgar demandas e necessidades em tecnologia assistiva para acesso de pesquisadores como forma de fomentar a produção de novas TA's. Outra proposta do banco é que a própria pessoa com deficiência, a partir de suas necessidades, possa cadastrar demandas no Observatório.

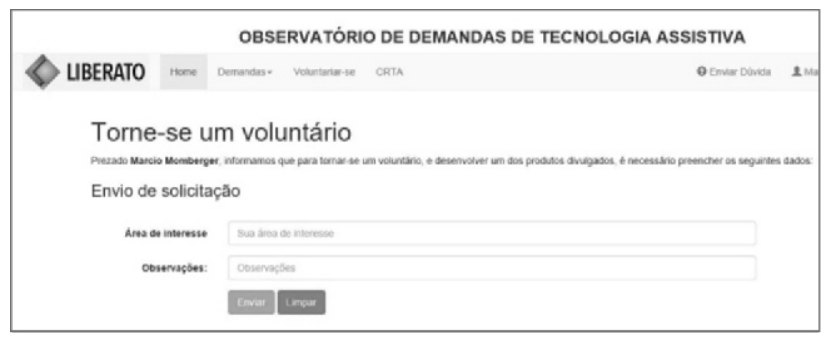

Figura 2. Tela do Observatório de demandas em TA Cadastro como estudante, pesquisador ou profissional voluntário.

O acesso é aberto e é possível se cadastrar como estudante ou pesquisador voluntário, adotando as demandas do banco para desenvolver pesquisa e proposta de solução. O banco está organizado da seguinte forma: demandas em TA; TA's em desenvolvimento e TA's desenvolvidas. O Observatório de demandas está disponível no site http://www.liberato.com.br/institucional/crta.

Importante ressaltar que o banco foi proposto pelas autoras e desenvolvido por uma aluna e dois professores do curso de Informática para Internet, da
Fundação Liberato. Esta proposta diferencia-se das demais por focar em problemas de pesquisa e demandas para o desenvolvimento de produtos e soluções me tecnologia assistive. Seu público-alvo são pesquisadores e emrpesários interessados em desnevolver soluções em TA, bem como a própria pessoa com deficiência interessada em alguma solução específica.

A Fundação Liberato integra-se à vida comunitária da região onde está localizada como polo irradiador do conhecimento científico-tecnológico, motivando os professors e alunos de seus cursos técnicos a desenvolverem soluções que contribuam com o bem comum. Nesse contexto, e observado um grande envolvimento dos profissionais e alunos da instituição com pesquisas na área da acessibilidade, investiu-se esforços para a criação de um Centro de Referência com foco na pesquisa, proposição e produção de tecnologia assistiva. O Centro possui parceria com diferentes universidades, empresas e entidades para o desenvolvimento de pesquisas e soluções tecnológicas na área de TA, tais como a aqui apresentada.

\section{CONSIDERAÇÕES FINAIS}

A pesquisa buscou identificar demandas de tecnologia assistiva, a partir de levantamento junto a professores de AEE. A investigação foi realizada em escolas públicas dos municípios de Novo Hamburgo e de São Leopoldo, no Rio Grande do Sul - Brasil. Os resultados apontaram que a maioria das demandas indicadas está relacionada a tecnologias que auxiliem na aprendizagem dos sujeitos atendidos pelos professores participantes da pesquisa. Esses professores prestam atendimento em espaços denominados Salas de Recursos Multifuncionais, um serviço de apoio pedagógico para a inclusão escolar em escolas de educação básica. Dos sete mais citados, três são direcionados a potencializar a aprendizagem das diferentes áreas do conhecimento; dois são para 
acessibilidade física; um para estimular a fala e um para auxiliar os movimentos manuais.

Além das demandas, observou-se que a maior parte das instituições pesquisadas cumpre o previsto na legislação, no que tange ao atendimento educacional especializado. No entanto, há indicativos da necessidade de maior apoio para promover acessibilidade das pessoas com deficiência na escola, tanto em termos de softwares e computacionais e de comunicação alternativa, quanto de materiais de adaptação física, o que pode ser suprido por meio da ampliação de TA nas escolas.

A pesquisa ainda mostrou que alguns dos espaços pesquisados possuem menos recursos materiais, uma vez que o atendimento educacional especializado, embora previsto em lei, está ainda em processo de implementação em determinados espaços, diferenciando-se por diversos motivos, dentre os quais destacam-se mobilizações da gestão da escola, a iniciativa do próprio professor da SRM.

\section{REFERÊNCIAS}

Bersch. R. (2015). Introdução à Tecnologia Assistiva. Porto Alegre. Disponível em www.assistiva.com.br. Acessado em 30 jun. 2015.

Casa Civil. Lei 12.796. De 04 de abril de 2013.

Casa Civil. Estatuto da Pessoa com deficiência. Lei № 13.146, de 6 de julho de 2015. Disponível em http://www.planalto.gov.br/ccivil_03/_Ato20152018/2015/Lei/L13146.htm. Acesso em agosto, 2015. Conselho Nacional de Educação. (2009). Resolução no 4 de 02 de outubro de 2009. Institui as Diretrizes operacionais para o atendimento educacional especializado na Educação Básica, modalidade da Educação especial. Conselho Nacional de Educação: Brasília.

Garcia, J. C, Passoni, I. R. (2008). Tecnologia Assistiva nas Escolas. São Paulo: Instituto de Tecnologia Social. MEC. (1996). Lei de Diretrizes a Bases da Educação Nacional-LDBEN 9394/96. Brasília.

MEC/SEESP. (2008). Política Nacional de Educação Especial na Perspectiva da Educação Inclusiva. Documento elaborado pelo Grupo de Trabalho nomeado pela Portaria Ministerial no 555, de 5 de junho de 2007, prorrogada pela Portaria no 948, de 09 de outubro de 2007. In: Inclusão. Educação Especial, 4(1), 7-17.

Ministério da Educação. (2011). Decreto no 7.611, de 17 de novembro de 2011. Dispõe sobre a educação especial, o atendimento educacional especializado e dá outras providências. Brasília.

Ministério da Educação. (2010). Nota Técnica SEESP/GAB/No 11/2010. Orientações para a institucionalização da Oferta do Atendimento Educacional Especializado - AEE em Salas de Recursos Multifuncionais, implantadas nas escolas regulares. Brasília. Disponível em 
http://portal.mec.gov.br/index.php?option=com_do $\mathrm{cman} \&$ view=download\&alias=5294-notatecnican 112010 \& category_slug = ma i o- $2010-$ pdf\&Itemid=30192. Acesso em 25 de setembro de 2015.

OMS. Organização Mundial da Saúde. (2012). Relatório mundial sobre a deficiência. World Health Organization. The World Bank. Tradução Lexicus Serviços Linguísticos. São Paulo: SEDPcD. Disponível em http://goo.gl/CiMUrW. Acessado em 27 Mai. 2015 (p. 247).

Secretaria de Direitos Humanos. Secretaria Nacional de Promoção dos Direitos da Pessoa com Deficiência (SNPD). SDH/SNPD. (2011). Viver Sem Limite. Plano
Nacional dos Direitos da Pessoa com Deficiência. Decreto 7.612, de 17 de novembro de 2011.

Subsecretaria Nacional de Promoção dos Direitos da Pessoa com Deficiência. (2009). B823 t Comitê de Ajudas Técnicas Tecnologia Assistiva. p.138. Brasília: CORDE. Disponível em

http://www.pessoacomdeficiencia.gov.br/app/sites/ default/files/publicacoes/livro-tecnologiaassistiva.pdf. Acesso em 12 agosto, 2014. 